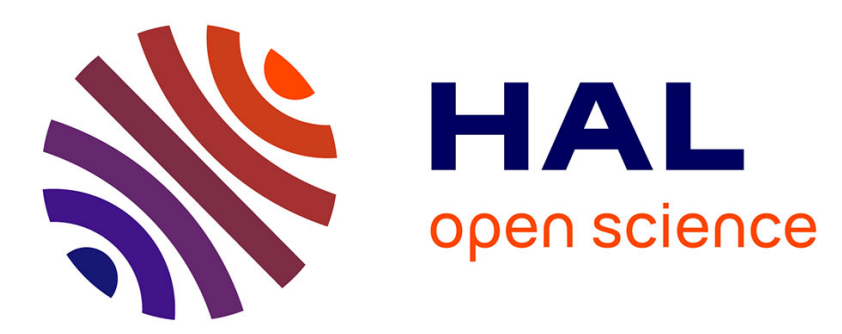

\title{
Equilibrium Degradation Levels in Irradiated and Pumped Erbium-Doped Optical Fibers
}

\author{
Franck Mady, Jean-Bernard Duchez, Yasmine Mebrouk, Mourad \\ Benabdesselam
}

\section{- To cite this version:}

Franck Mady, Jean-Bernard Duchez, Yasmine Mebrouk, Mourad Benabdesselam. Equilibrium Degradation Levels in Irradiated and Pumped Erbium-Doped Optical Fibers. IEEE Transactions on Nuclear Science, 2015, 62 (6), 10.1109/TNS.2015.2480888 . hal-01223245

\section{HAL Id: hal-01223245 \\ https://hal.science/hal-01223245}

Submitted on 2 Nov 2015

HAL is a multi-disciplinary open access archive for the deposit and dissemination of scientific research documents, whether they are published or not. The documents may come from teaching and research institutions in France or abroad, or from public or private research centers.
L'archive ouverte pluridisciplinaire HAL, est destinée au dépôt et à la diffusion de documents scientifiques de niveau recherche, publiés ou non, émanant des établissements d'enseignement et de recherche français ou étrangers, des laboratoires publics ou privés. 


\title{
Equilibrium Degradation Levels in Irradiated and Pumped Erbium-Doped Optical Fibers
}

\author{
Franck Mady, Jean-Bernard Duchez, Yasmine Mebrouk and Mourad Benabdesselam
}

\begin{abstract}
This work investigates the local interplay between radiation-induced darkening and pump-induced bleaching in erbium-doped silica optical fibers. Thanks to proper characterization conditions using short fiber samples, we demonstrate that degradation levels can be tuned reversibly between equilibrium levels by varying the pump power and/or the dose rate while the dose is being accumulated. This novel finding inspires a local physical model of the radiation-induced degradation of erbium-doped fibers in active conditions. This model is shown to reproduce all empirical features, including dose rate and pump power dependencies. This validated local model could be implemented in long fiber amplifier simulations.
\end{abstract}

Index Terms-Erbium-doped fiber amplifiers, Modeling, Optical fibers, Radiation effects, Radiation-induced attenuation.

\section{INTRODUCTION}

$\mathrm{E}$ rbium-doped fibers amplifiers (EDFA) operating around $1.55 \mu \mathrm{m}$ have proved to be a reliable technology, notably in ground fiber telecommunication networks. They also represent an attractive option to design compact and efficient optical amplifiers to be used in the harsh space environment, as in satellite fiber optic gyroscopes (FOG) or in power fiber laser sources required for future optical inter-satellite links (OISL) or remote sensing (LIDAR). The development of such systems is though limited by the fact that erbium-doped silicabased fibers (EDF) are damaged by ionizing radiations. An excess optical loss indeed builds-up due to the formation of color centers upon ionization effects, this effect being referred to as 'darkening'. A rather significant amount of publications has addressed the radiation-resistance of EDF and EDFA for more than 20 years [1-13], and several technological routes have been shown to greatly improve their radiation tolerance. Hardening recipes are based on cerium co-doping [12,13] (the benefit of cerium has been known for at least 40 years [14]), on the drastic reduction of aluminum content through nanoparticle doping process [11] (aluminum greatly enhances the formation of color centers), or on hydrogen loading coupled with hermetic fiber coating $[7,8,12,13]$. Thanks to this knowledge, EDF-based FOG have been flying around the

Manuscript received 07/06/2015.

This work falls within the framework of the HAPoLO (098-A011-2408) and PARADYSIO (ANR-11-JS04-007) projects supported by the AirbusGroup Foundation and the French Research National Agency (ANR).

F. Mady, J-B. Duchez, Y. Mebrouk and M. Benabdesselam were with the University of Nice Sophia Antipolis, Laboratoire de Physique de la Matière Condensée, CNRS UMR 7336, Faculté des Sciences, Parc Valrose, 06108 Nice, France (e-mail: franck.mady@unice.fr).
Earth already. However, mechanisms of the radiation-induced darkening of EDF remain unclear. Whereas it is widely admitted that RIA is related to the host matrix absorption and color centers, some basic questions are still not clearly elucidated: what is the exact nature of color centers? What are the intrinsic silica- or dopant-related defects acting as precursors? How do precursors compete as regards trapping of charge carriers? How do color centers interact with the lights that propagate in the fiber? What are their thermal activation and photo-ionization energies? Etc... Such precise questions are crucial to gain the detailed understanding required to introduce relevant prediction/explanation frameworks (modeling). Models have been tentatively proposed to account for the dose-rate and total dose degradation dependence $[3,10]$, but they rely on semi-empirical descriptions (power law dependence of the color center generation rate) and therefore lack in physical basis. In addition, a most basic mitigation agent, namely the pump, has not received the attention it deserves. It is well known that light at pump wavelength (most often around $980 \mathrm{~nm}$ ) is responsible for photo-bleaching (PB) of darkened EDF [8,15], but pump power is too often not specified or not taken into account in data interpretation. A proper analysis of the pump-induced PB is however of crucial importance, first because the degradation of EDF is generally assessed by the measurement of the socalled radiation-induced attenuation (RIA) at pump or signal wavelengths, which requires of course the guidance of a light that does not act as a probe only, and second because the pump is naturally present in active operation conditions. Actually, the 1 to $10 \mathrm{~m}$ EDF lengths used in most of cited studies are a pitfall for PB characterization. They are chosen to comply with operation conditions, but result in an inhomogeneous distribution of the pump power along the fiber and so in a distributed PB efficiency that makes the pump effect - if sought through the length-integrated RIA - highly extrinsic (length-dependent). Therefore realistic amplifier lengths do not offer convenient conditions to investigate properly the sole role of $\mathrm{PB}$. In what follows, we use short fiber pieces to demonstrate new remarkable features in the interplay between pump-induced $\mathrm{PB}$ and radiation-induced darkening. Highlighted mechanisms are included in a physical model of the local EDF darkening, as a function of the dose rate and pump power. This model is validated against data.

\section{EXPERIMENTAL}

The measurement setup, sketched in Fig. 1, is similar to the 
one presented in [16], except that the $633 \mathrm{~nm}$ probe laser is not used here. The fiber under test (FUT) is in-core pumped at 976 $\mathrm{nm}$ (temperature and fiber-Bragg grating stabilized monomode laser diode) while being homogeneously irradiated in the narrow beam of an X-ray generator (Inel XRG 3500, copper anode, $45 \mathrm{kV}$ accelerating voltage). The FUT is spliced to passive single mode fibers (SMF) at both ends. A large $3 \mathrm{~mm}$ thick lead lid, in which a rectangular opening can be adjusted to the FUT length, protects both SMF and splices. This lid efficiently screens soft x-rays and makes unwanted losses that could appear in splices and SMF negligible compared to the RIA of the FUT. Two InGaAs photodiodes served as powermeters (PM1 and PM4) to monitor launched and transmitted pump powers. Records made with PM1, which approximately corresponds to $1 \%$ of the power supplied by the laser diode, are properly corrected from losses in the SMF, multiplexor and in the connecting splice to provide the actual power launched in the FUT (pump power indicated below well correspond to actual values). Dose rates given to the FUT are monitored by an ionization chamber (PTW 23342 with an UnidosE electrometer) placed in the beam, right below the FUT. The 976 nm RIA is calculated according to:

$$
\text { RIA }_{976}=-\frac{10}{L \times \ln (10)} \ln \left(\frac{P_{\text {out }}(t)}{P_{\text {in }}(t)} \times \frac{P_{\text {in }}(0)}{P_{\text {out }}(0)}\right) \text {, in } \mathrm{dB} \mathrm{m}^{-1} .
$$

In this relationship, $L$ is the FUT length in $\mathrm{m}, P_{\mathrm{in}}(t)$ and $P_{\text {out }}(t)$ are input and output powers at time $t$, respectively. $P_{\text {in }}$ is actually constant $\left(P_{\text {in }}(t)=P_{\text {in }}(0)\right.$, fluctuation limited to $\left.2 \%\right)$.

We investigated a commercial off-the-shelf (COTS), Er804/125, and a tailor-made alumino-silicate EDF, M04, fabricated and drawn in our laboratory by the standard MCVD and solution doping techniques. The Er80-4/125 fiber is commercialized by Liekki (n-light) as a very highly doped erbium fiber designed for fiber lasers and amplifiers (80 $\mathrm{dB} \mathrm{m}^{-1}$ at $1530 \mathrm{~nm}$, erbium ion concentration $3 \times 10^{19} \mathrm{~cm}^{-3}$ ). The $1530 \mathrm{~nm}$ absorption of the M04 fiber, designed to have a rather similar erbium content, has been measured to $72.3 \mathrm{~dB} \mathrm{~m}^{-1}$ (erbium ion concentration $2.8 \times 10^{19} \mathrm{~cm}^{-3}$ ). We used very short fiber pieces to avoid amplification of spontaneous emission (ASE) and ensure a uniform distribution of both pump power and inversion ratio of $\mathrm{Er}^{3+}$ ions before irradiation. For instance, the pump power was always virtually constant throughout $2 \mathrm{~cm}$-long samples (varying by less than $0.8 \%$ ). In long fibers, ASE powers are not negligible and their profiles, together with those of pump power, excitation ratio, and therefore PB efficiency, strongly depend on $L$ and $P_{\text {in }}$. By taking short fiber pieces, we free the problem from extrinsic spatial variations to retain a well-defined power as the unique pump parameter. Profile effects, that additionally accompany changes in $P_{\text {in }}$ or $L$ and are necessarily integrated when the RIA is averaged over several meters, are thus ruled out. Builtin losses from the $976 \mathrm{~nm}$ laser diode to PM4, deduced from initial attenuation (in the presence of a FUT but before RIA develops), is about $3.05 \mathrm{~dB}$ in all experiments.

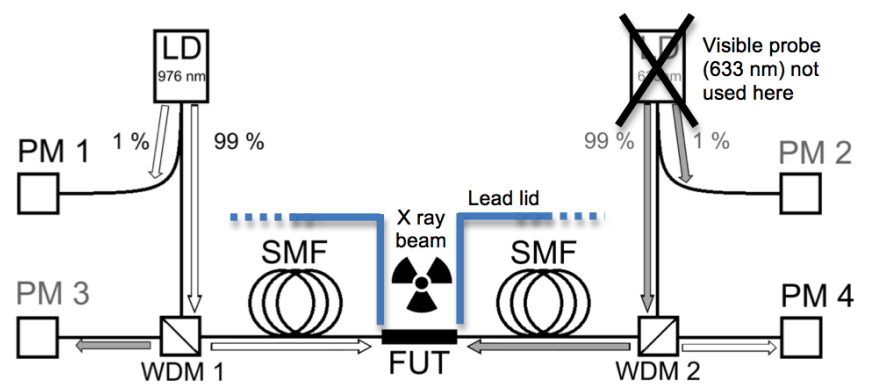

Fig. 1. Experimental setup, similar to that of reference [16] except that the visible RIA probe at $633 \mathrm{~nm}$ is not used in this study. PM are power-meters, SMF are passive single mode fibers (commercial SM980) and WDM are wavelength division multiplexors. A lead lid protects splices and SMF.

\section{RESULTS}

A typical experiment is reported in Fig. 2 for the Er80 fiber. A unique $L=2 \mathrm{~cm}$-long fiber sample has been irradiated and pumped continuously for almost 3 days. The dose rate $D^{\prime}$ and the pump power $P_{\text {in }}$ have been switched between various constant values, as indicated in graphs. The upper part of the figure displays the evolution of the RIA at $976 \mathrm{~nm}$ whereas the lower part gives the cumulated ionizing dose. The experiment can be divided into 3 parts: A, B and C. In A, the pump power was constant $\left(P_{\text {in }}=132 \mathrm{~mW}\right)$. The dose rate has been first set at $58 \mathrm{krad} / \mathrm{min}$ and then decreased by steps down to 39,20 , $10.5,5,2.6,1.3$ and $0 \mathrm{krad} / \mathrm{min}$ before being raised again to 5 and $10.5 \mathrm{krad} / \mathrm{min}$. In the $\mathrm{B}$ region, the dose rate was constant $\left(D^{\prime}=10.5 \mathrm{krad} / \mathrm{min}\right)$ but $P_{\text {in }}$ was switched from 132 to 167.8 $\mathrm{mW}, 48.2 \mathrm{~mW}$, and raised back to $132 \mathrm{~mW}$. The pump power was again dropped to $48 \mathrm{~mW}$ to enter the $C$ region where we started with $D^{\prime}=0$ before switching to 20,39 , and $58 \mathrm{krad} \mathrm{min}^{-1}$. Fig. 3 reports the record of another 6-day irradiation and pumping sequence for the same fiber $(\operatorname{Er} 80)$ but a new sample of length $L=0.5 \mathrm{~cm}$. Cumulated doses at the end of the various steps are now indicated in the RIA graph. This experiment is divided is 5 regions (A-E), each being characterized by a fixed input pump power $(74.7-178.5 \mathrm{~mW}$ ) and a similar sequence of decreasing dose rates in the 55-10 $\mathrm{krad} \mathrm{min}^{-1}$ range. Regions $\mathrm{A}$ and $\mathrm{C}$ include long photobleaching periods, of about 2 and 3 days respectively. The fiber sample is then submitted to the pump only (no x rays), at the same power $(132 \mathrm{~mW})$, but starting from different degradation levels (see arrows). Fig 4 displays the result of a similar 10-hour experiment made on a $2 \mathrm{~cm}$-long sample of our laboratory-made M04 fiber. RIA of Fig. 2 to 4 show positive or negative short overshoots that correspond to natural transients betraying rapid pump power changes (switches). Positive overshoots mark sudden power rises whereas negatives ones reveal power drops. In these figures, the RIA is always calculated relative to the initial fiber absorption at $976 \mathrm{~nm}$ before irradiation, which is mainly due non-inverted erbium ions. Since the first applied pump power is always $132 \mathrm{~mW}$, displayed RIA are constantly estimated with respect to the pristine absorption at $132 \mathrm{~mW}$, which is much lower than the nominal small signal absorption for our 2 and $0.5 \mathrm{~cm}$-long samples (about $0.39 \mathrm{~dB} \mathrm{~m}^{-1}$ pristine loss at $976 \mathrm{~nm}$ and $132 \mathrm{~mW}$, the small signal value being close to 30 $\mathrm{dB} \mathrm{m} \mathrm{m}^{-1}$ at this wavelength for the Er80-4/125 fiber). If the 


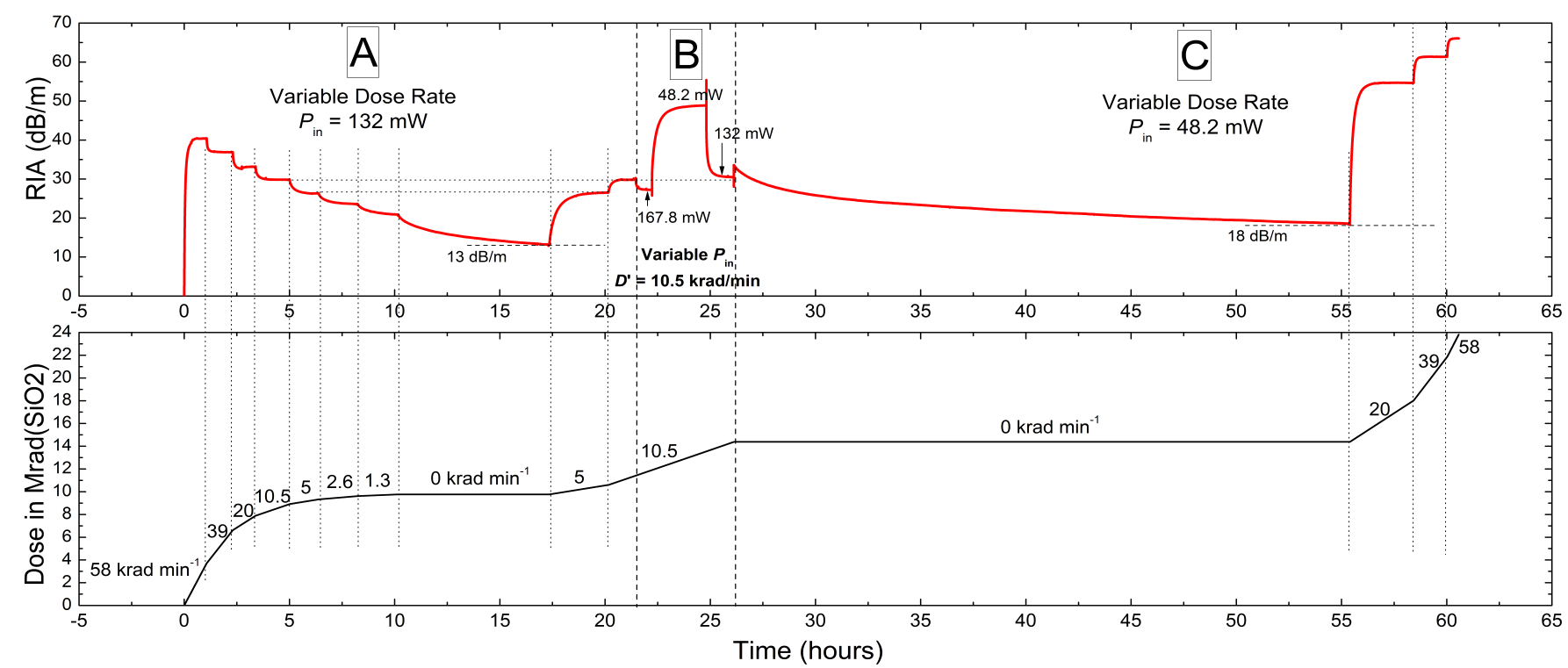

Fig. 2. Temporal evolution of the RIA under various pump power/dose rate couples (upper part), and the corresponding evolution of the cumulated dose in silica (lower part), applied continuously on the same $2 \mathrm{~cm}-$ long piece of the COTS Er80-4/125 fiber.

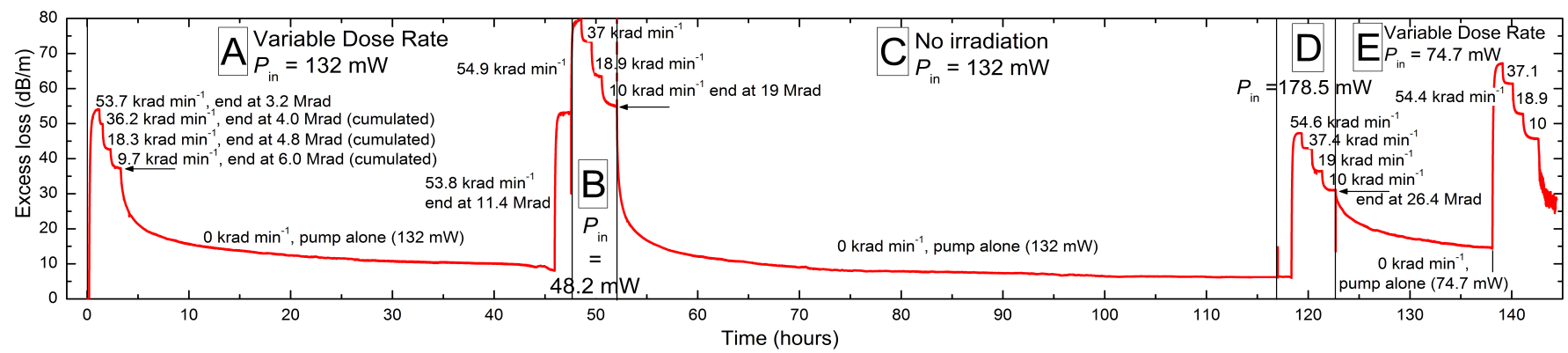

Fig. 3. Temporal evolution of the RIA under various pump power/dose rate (indicated on graph) applied continuously on the same $0.5 \mathrm{~cm}-\mathrm{long}$ piece of the COTS Er80-4/125 fiber. Cumulated doses reached at the end of each step are indicated.

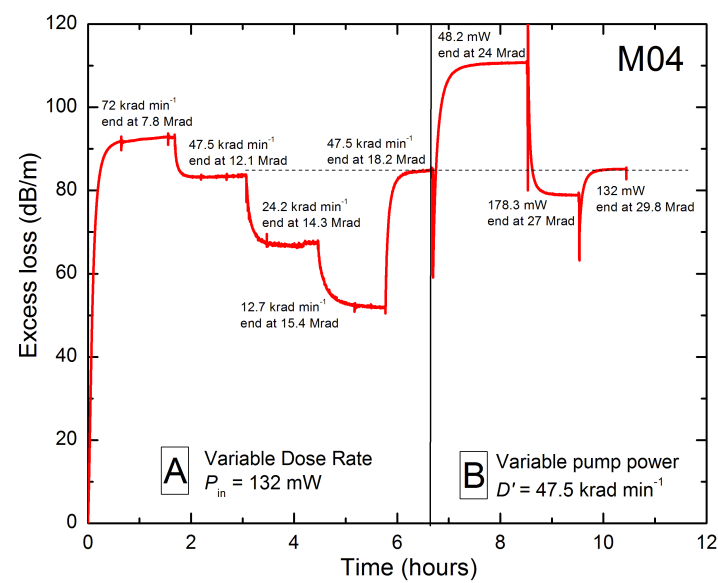

Fig. 4. Temporal evolution of the RIA under various pump powers/dose rates (indicated on graph) applied continuously on the same $2 \mathrm{~cm}$-long piece of the M04 laboratory-made EDF. Cumulated doses reached at the end of each step are indicated.

pristine absorption is still virtually equal to $0.39 \mathrm{~dB} \mathrm{~m}^{-1}$ for pump powers $>132 \mathrm{~mW}$, it can be perceptibly greater at pump powers $<132 \mathrm{~mW}\left(1.05 \mathrm{~dB} \mathrm{~m}^{-1}\right.$ at $\left.48.2 \mathrm{~mW}\right)$. As a result, RIA of Fig 2-4 are slightly overestimated at pump powers below $132 \mathrm{~mW}$, but the error is lower than $1 \mathrm{~dB} \mathrm{~m}^{-1}$ for the lowest power we used $(48.2 \mathrm{~mW})$.

\section{A. Equilibrium degradation levels (EDL)}

In experiments of Fig. 2-4, each couple $\left(P_{\text {in }}, D^{\prime}\right)$ was maintained a sufficiently long time to demonstrate that the RIA can reach a steady-state level (hereafter termed equilibrium degradation level, EDL). This original finding, made possible by the use of short EDF samples, well illustrates that a pump-induced PB effect exists. PB competes with radiation-induced darkening and balances it after a transient evolution, as confirmed by the fact that EDL are raised when the dose rate is increased and/or pump power is decreased. After its initial steep increase, the RIA decreases in zone A of Fig. 2-4 (transmission recovery) while the total dose increases continuously! This uncommon behavior is obviously enabled by PB, but it would not be observed if the dose rate was switched before EDL are reached. EDL formed under irradiation are entirely determined by the couple $\left(P_{\mathrm{in}}, D^{\prime}\right)$. They neither depend on the cumulated dose, i.e. the exposure history of the sample, nor on the starting RIA level. For instance, the couple $(132 \mathrm{~mW}, 10.5 \mathrm{krad} / \mathrm{min})$ is formed twice in region $\mathrm{A}$ and once in region $\mathrm{B}$ of Fig. 2, from various total doses and different initial RIA levels, but it always results in the same EDL around $30 \mathrm{~dB} \mathrm{~m}^{-1}$ despite a $>20 \mathrm{~h}$ temporal gap. In Fig. 2 and 4, dashed horizontal lines connect EDL obtained at identical pump/dose rate couples. The fact that existence and properties of EDL are established for two 
commercial and tailor-made EDF, fabricated in different units, probably with different co-dopants indicates that they result from most basic mechanisms (the unknown Er80 fiber composition is probably based on a alumino-silicate matrix also, even if additional co-dopants are present).

\section{B. Zero-dose rate EDL}

EDL obtained in the absence of irradiation are particular in some respects. First, they appear after very long transient periods, lower pump powers leading to longer transients (see e.g. photo-bleaching periods in regions $\mathrm{A}$ and $\mathrm{C}$ of Fig. 2). Much swifter kinetics, dominated by the dose rate, is achieved under irradiation. Second, they remain equal to zero when the pump is applied on a pristine sample. Indeed, EDF are not subjected to photo-darkening (PD) like ytterbium-doped fibers [16]. We submitted for instance a fresh $2 \mathrm{~cm}$-long sample of Er80 to PD by pumping at $132 \mathrm{~mW}$ for more than 64 hours without observing any noticeable photo-induced loss. In the absence of irradiation, non-zero EDL are only reached when starting from some previous RIA level, i.e. after a certain dose (case of photo-bleaching, Fig. 2 and 3), and in that sense zerodose rate EDL depend on the initial darkening state. However, photo-bleaching periods followed at $P_{\text {in }}=132 \mathrm{~mW}$ in regions $\mathrm{A}$ and $\mathrm{C}$ of Fig. 3, which start from different RIA levels but tend towards the same EDL around $6 \mathrm{~dB} \mathrm{~m}^{-1}$, show that zerodose rate EDL are independent of the initial degradation state provided it is not zero. The increase of the pump power from 132 to $178.5 \mathrm{~mW}$ at the onset of region $\mathrm{D}$ (between $t \sim 117$ and $118.5 \mathrm{~h}$ in Fig. 3) does not lead to further decrease of this EDL. Therefore, zero-dose rate EDL neither depend on the pump power. This conclusion could not be drawn from photobleaching periods reported in Fig. 2, because of the very long transient at $P_{\text {in }}=48.2 \mathrm{~mW}$ (region C) which is not completed when $\mathrm{x}$ rays are turned on again (at $t \sim 55 \mathrm{~h}$ ). In this case, the zero-dose rate EDL is expected to be slightly below 13 $\mathrm{dB} \mathrm{m} \mathrm{m}^{-1}$. The fact that photo-bleaching does not decrease the RIA down to zero but to a zero-dose rate EDL independent of the pump power and of the initial RIA level strongly suggest that part of color centers is not bleachable.

\section{EDL dependence on dose rate and pump power}

EDLs extracted from Fig. 2 to 4 are plotted in Fig. 5 (resp. 6) as a function of the dose rate (resp. pump power) at fixed pump powers (resp. dose rate).

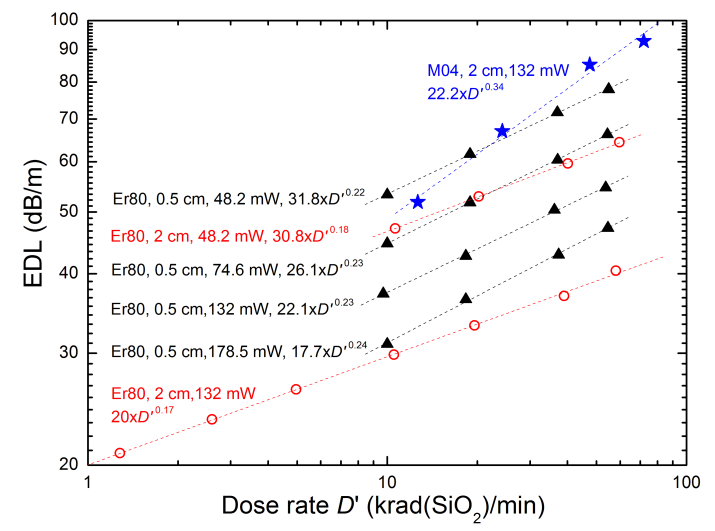

Fig. 5. EDL as a function of dose rate at fixed pump powers between 48.2 and $178.5 \mathrm{~mW}$, and power law fits in dashed lines. Fiber types, sample lengths, pump powers and fit equations are indicated on graphs.

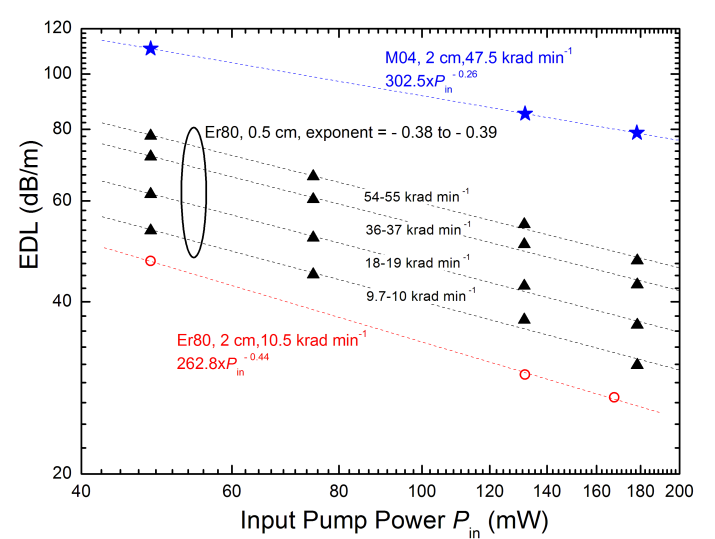

Fig. 6. EDL as a function of input pump power at fixed dose rates between 10 and $55 \mathrm{krad} \mathrm{min}^{-1}$, and power law fits in dashed lines. Fiber types, sample lengths, dose rates and fit equations or exponents are indicated on graphs.

EDL variations are all well fitted by power laws in $D^{a}$ or $P_{\text {in }}{ }^{b}$. For a given fiber and length, the dose rate exponent $a$ is quite independent of pump power. For Er80 samples, $a=0.22-0.23$ for $L=0.5 \mathrm{~cm}$, and $0.17-18$ for $L=2 \mathrm{~cm}$ (Fig. 5). Conversely, the pump power exponent $b$ lies between -0.38 and -0.39 whatever the dose rate for $\operatorname{Er} 80$ and $L=0.5 \mathrm{~cm}$ and its value decreases slightly down to -0.44 when $L=2 \mathrm{~cm}$. Variations in $a$ and $b$ values are more pronounced when passing from the Er80 to the M04 fiber. For $L=2 \mathrm{~cm}$ and $P_{\text {in }}=132 \mathrm{~mW}$, the dose rate exponent of M04 is for instance twice that of $\operatorname{Er80}(a$ $=0.34$ instead of 0.17 , see Fig. 5). The higher sensitivity of $a$ and $b$ to the fiber type, by comparison to extrinsic parameters such as dose rate, pump power or length, may indicate that these exponents primarily reflect intrinsic mechanisms whose efficiency vary with composition or doping level. A power law dependence of the RIA on the dose rate $D^{\prime}$ is not - a priori - an original finding, since the RIA was empirically found to vary as $D^{,(1-f)} D^{f}$, where $D$ is the dose and $f$ an exponent $[3,4,17]$ ( $f$ is close to 0.8 in [4]). However, we deal here with new reference levels, i.e. EDL, which do not depend on the dose (RIA is frozen at the EDL, even under continuous irradiation at constant $P_{\text {in }}$ and $D^{\prime}$ ). We have therefore $\operatorname{EDL} \propto\left(D^{\prime a} \times P_{\text {in }}{ }^{b}\right)$, the dose exponent being zero.

\section{Intermittent Pumping}

Stabilization of the RIA at EDL is a direct consequence of continuous pumping. The modification of this property in case of intermittent pumping is now addressed, for two reasons. First, intermittent pumping can be considered more representative of actual operation conditions in space. To save energy, the pump should be turned on at some required times only (when the EDFA-based system is needed) rather than continuously. Since $976 \mathrm{~nm}$ photons do not only probe RIA but also affect it, it is also necessary to limit pump ignition to the bare minimum (discrete measurements) and assess whether the 'intrinsic' $976 \mathrm{~nm}$ RIA can be estimated reliably.

Intermittent pumping tests were made on fresh $2 \mathrm{~cm}$-long pieces of the Er80 fiber at various dose rates. Results are illustrated in Fig. 7. The lower plot has been obtained under 40, 60 and $10 \mathrm{krad} \mathrm{min}^{-1}$ continuous irradiations, by turning on the pump at $132 \mathrm{~mW}$ for $5 \mathrm{~s}$ every 15 minutes (pump ignition time ratio $0.56 \%)$, or every hour $(0.14 \%)$. We set the record frequency to get one data point per second. RIA levels are 
higher than EDL found in Fig. 2 at this dose rates and pump power $\left(40,36\right.$ and $30 \mathrm{~dB} \mathrm{~m}^{-1}$ respectively), but the pump effect is still strong. It prevents the RIA from continuous increase and leads to its stabilization at a mean level around $100-120 \mathrm{~dB} \mathrm{~m}^{-1}$ at the three dose rates and both pump ignition periods. We again reduced the pump impact by dropping the pump power down to $7.7 \mathrm{~mW}$ and the ignition time ratio to 0.06 and $0.03 \%$ ( $2 \mathrm{~s}$ ignition every 1 or 2 hours). We then obtained the upper plot of Fig. 7 at 40 and $60 \mathrm{krad} \mathrm{min}^{-1}$ dose rates. Despite this further reduction, PB is still sufficient to make the RIA stabilize, now at a mean level around 260-270 $\mathrm{dB} \mathrm{m}{ }^{-1}$. In intermittent pumping conditions, the EDL still exists under the form of mean steady-state levels. Such mean EDL tend to increase with the dose rate, but very slowly compared with the case of continuous pumping (at least within the 10-60 krad $\mathrm{min}^{-1}$ range explored in Fig. 7). Mean EDL are markedly enhanced at low pump power. They are almost independent of the ignition period (time between two successive pump ignitions), but highly sensitive to the duration of the pump operation. The RIA drops indeed very rapidly upon pump lighting, as evidenced by steep falls in Fig. 7. The fall rate tremendously increase with the starting RIA level: about $28 \mathrm{~dB} \mathrm{~m}^{-1} \mathrm{~s}^{-1}$ around the mean EDL at 270 $\mathrm{dB} \mathrm{m}^{-1}$ (upper plot at $7.7 \mathrm{~mW}$ ), but 'only' $14 \mathrm{~dB} \mathrm{~m}^{-1} \mathrm{~s}^{-1}$ around $110 \mathrm{~dB} \mathrm{~m}^{-1}$ (lower plot at $132 \mathrm{~mW}$ ) for a pump power which is though $>14$ times greater. To maintain a low RIA, it is therefore much more efficient to increase the pump operation time than its ignition frequency. Even short, low-frequency and low-power pump actions induce efficient RIA mitigation. As a result, measuring the 'intrinsic' RIA at $976 \mathrm{~nm}$ turns out to be a really hard task! At the very low dose rates of spacebased applications, RIA will develop very slowly and will not be restored significantly between successive pump operations. Successive ignitions should keep degradation at a low level provided their duration and period are properly optimized. We finally estimated the room temperature annealing (RTA) by stopping $\mathrm{x}$-rays and waiting for $9 \mathrm{~h}$ before the last RIA record. RTA is more significant when it starts from the $270 \mathrm{~dB} \mathrm{~m}^{-1}$ level (upper plot, almost - $40 \%$ in $9 \mathrm{~h}$ ). The relative annealing is 4 times smaller when starting from $90 \mathrm{~dB} \mathrm{~m}^{-1}$ (lower plot). In Fig. 2 to 4, RIA levels were such that RTA was weak: with significant RTA, we could not reach the same EDL, as we did three times in $21 \mathrm{~h}$ at $132 \mathrm{~mW}$ and $10.5 \mathrm{krad} \mathrm{min}^{-1}$ (Fig. 2).

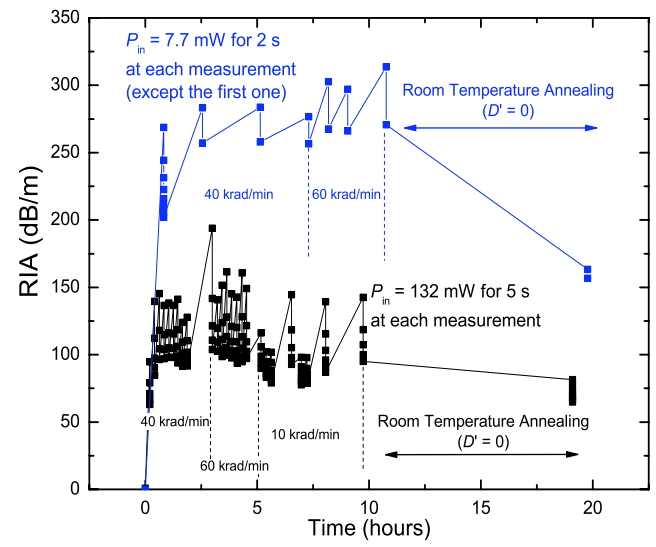

Fig. 7. RIA at $976 \mathrm{~nm}$ measured by short pump ignition periods. Lower plot: $P_{\text {in }}=132 \mathrm{~mW}$ during $5 \mathrm{~s}$ every $15 \mathrm{~min}$ or $1 \mathrm{~h}$. Upper plot: $P_{\text {in }}=7.7 \mathrm{~mW}$ during $2 \mathrm{~s}$, pump ignition period $=1$ or $2 \mathrm{~h}$ at $60 \mathrm{krad} \mathrm{min}^{-1}$ and $2 \mathrm{~h}$ at $40 \mathrm{krad} \mathrm{min}^{-1}$.

\section{MODELING AND DOSE RATE EXTRAPOLATION}

\section{A. Local physical model}

The physical model of EDF degradation is adapted from that proposed for ytterbium-doped fibers (YDF) in [18] by introducing two specificities, namely the absence of photodarkening and the existence of a non-bleachable RIA. We also already proposed that radiation-induced ionization effects, which start by electron-hole pair excitation, are somewhat similar in YDF [19] and EDF [20]. Both notably include reduction of $\mathrm{Yb}^{3+}$ or $\mathrm{Er}^{3+}$ ions into divalent ions (due to free electron capture), and trapping of free holes on silica- or dopant-related precursors to form color centers. Spectrallyresolved thermoluminescence analyses, showing the typical emissions of trivalent ions, revealed that radiation-induced $\mathrm{Yb}^{2+}$ or $\mathrm{Er}^{2+}$ ions are the dominant recombination centers when trapped holes are released by thermal annealing (recombination re-forming trivalent ions) [19,20]. These hypotheses inspired the darkening/bleaching scheme of Fig. 8.

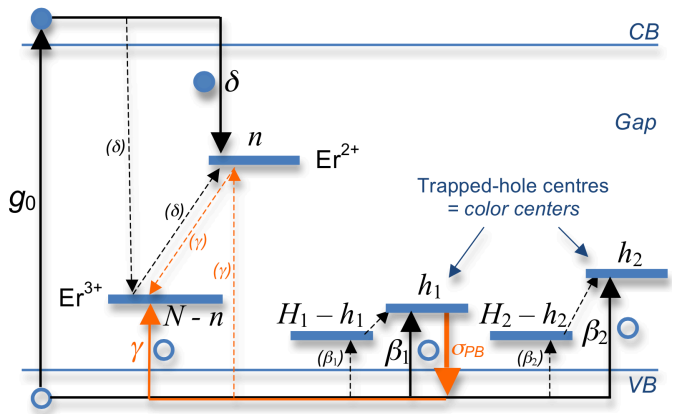

Fig. 8. Energy level scheme of the proposed model. Dashed arrows = trapping/recombination steps. Solid arrows $=$ effective energy transitions between initial and final states.

The ionizing radiation creates electron-hole pairs at a rate $g_{0}$, representative of the dose rate. The total ionizing dose thus corresponds to the total density of generated pairs given by $g_{0} \times t_{\text {irr }}$, where $t_{\text {irr }}$ is the irradiation time. Electrons injected in the conduction band (CB) can be trapped by $\mathrm{Er}^{3+}$ ions (initial density $N$ ) to form $\mathrm{Er}^{2+}$ ions in density $n$ ( $\delta$ transitions). Holes in the VB are trapped by gap states to form color centers $\left(H_{1,2}\right.$ precursor states occupied by $h_{1,2}$ holes, $\beta_{1,2}$ transitions). These gap states should be associated with defects of the host matrix, i.e. silica point defects or dopant-related centers (due to, e.g., aluminum, phosphorus...). We assume photo-bleaching (PB) results from the photo-ionization of trapped holes to the VB $\left(\sigma_{\mathrm{PB}}\right.$ transition) and from their subsequent recombination on $\mathrm{Er}^{2+}$ ions ( $\gamma$ transition). PB only concerns shallowest trappedhole states (density $h_{1}$ ). 'Optically disconnected' deep hole traps $\left(h_{2}\right)$ are introduced to account for the non-bleachable part of the RIA. Actually, even shallow traps are likely too deep to enable direct ionization by single pump photons $(1.27 \mathrm{eV})$, because efficient photo-ionization requires about twice the thermal activation energy which is typically around or greater than $1 \mathrm{eV}$. Three mechanisms could then contribute to PB: a two-pump photon absorption $(2.54 \mathrm{eV})$, the absorption of green photons emitted after pump-induced upconversion of $\mathrm{Er}^{3+}$ ions $\left({ }^{4} \mathrm{~S}_{3 / 2}\right.$ to ${ }^{4} \mathrm{I}_{15 / 2}$ radiative de-excitation, $\left.2.28 \mathrm{eV}\right)$, or cooperative de-excitation of 2 close inverted $\mathrm{Er}^{3+}$ ions upon spontaneous ${ }^{4} \mathrm{I}_{13 / 2}$ to ${ }^{4} \mathrm{I}_{15 / 2} 1530 \mathrm{~nm}$ relaxation $(2 \times 0.81 \mathrm{eV}=$ 
$1.62 \mathrm{eV})$. Retaining cooperative ion-pair de-excitation as the energy supplier of pump-induced $\mathrm{PB}$ enabled us to explain various experimental features in YDF [19]. In EDF however, first modeling attempts showed this hypothesis does not correctly render the observed EDL dependence on pump power. In EDFA operating at $1530 \mathrm{~nm}$, upconversion is a detrimental effect causing parasitic losses. It is normally weak (neglected in [3] for instance) at low or moderate pump powers. It probably contributes to $\mathrm{PB}$ at high power levels, but its consideration adds much complexity in the equations and their treatment. For simplicity, we suppose that two-photon absorption is the dominant source of PB. This rough limitation offers future refinement opportunities. Fig. 8 presents a minimal model including only two discrete hole-trapping levels. Other possible electron traps besides $\mathrm{Er}^{3+}$ ions are neglected, as well as thermal release in agreement with preceding conclusions. It would be more realistic to include a distribution of gap levels because of the variety of centers identified in silicate or alumino-silicate glasses and of the inhomogeneity of such materials. This would introduce a significant complexity that would again interfere with the validation of the crucial mechanisms that are basically sufficient to explain the observed behaviors.

Transitions of Fig. 8 can be rationalized by the set of coupled non-linear differential equations (1)-(5) defining the local darkening/bleaching evolution model.

$$
\begin{aligned}
\frac{d n_{\mathrm{c}}}{d t} & =g_{0}-\delta n_{\mathrm{c}}(N-n), \\
\frac{d n}{d t} & =\delta n_{\mathrm{c}}(N-n)-\gamma h_{\mathrm{v}} n, \\
\frac{d h_{1}}{d t} & =\beta_{1} h_{\mathrm{v}}\left(H_{1}-h_{1}\right)-\sigma_{\mathrm{PB}} P_{\mathrm{in}}^{2} N_{\mathrm{v}} h_{1}, \\
\frac{d h_{2}}{d t} & =\beta_{2} h_{\mathrm{v}}\left(H_{2}-h_{2}\right), \\
\frac{d h_{\mathrm{v}}}{d t} & =g_{0}-\beta_{1} h_{\mathrm{v}}\left(H_{1}-h_{1}\right)-\beta_{2} h_{\mathrm{v}}\left(H_{2}-h_{2}\right) \\
& +\sigma_{\mathrm{PB}} P_{\text {in }}{ }^{2} N_{\mathrm{v}} h_{1}-\gamma h_{\mathrm{v}} n .
\end{aligned}
$$

In (1)-(4), $n_{\mathrm{c}}$ and $h_{\mathrm{v}}$ are the densities of electrons in the CB and of holes in the $\mathrm{VB}$, respectively. $N_{\mathrm{V}}$ is the equivalent density of states in the VB. The photo-bleaching rate of shallow traps is taken proportional to the square of the pump power $P_{\text {in }}$, as usual for two-photon processes, and the proportionality coefficient is $\sigma_{\mathrm{PB}}$. The latter includes among others the photo-ionization cross-section of trapped-hole color centers. Capture and recombination coefficients of free carriers $\left(\beta_{1,2}, \delta\right.$ and $\left.\gamma\right)$ are commonly described as the product of the thermal velocity of free carriers in their band by the trapping or recombination cross section.

Color centers associated with shallow and deep trapped-hole states produce overlapping optical absorption bands in the near infra-red range so they both contribute to the $976 \mathrm{~nm}$ RIA. Calculations show that transient band populations $n_{\mathrm{c}}$ and $h_{\mathrm{v}}$ are always negligible (by far) so $n$ and $h_{1}+h_{2}$ are virtually identical. The computed RIA is proportional to the density of color centers, $h_{1}+h_{2}$ (or $n$ ), which is the output quantity to be compared with data. Time-resolved RIA and EDL have been simulated by solving (1)-(5) numerically with the Scilab® software (all densities being initially equal to zero).

\section{B. Validation against data}

We first simulated parts of reported experiments to check the capability of the model of reproducing basic experimental features. Comparison is relevant since we confront at this stage a local model, rigorously valid for a fiber length tending to zero, to data measured in very short fiber samples. Calculations have been made with the following material parameters: $N=10 \times\left(H_{1}+H_{2}\right)=3 \times 10^{19} \mathrm{~cm}^{-3}$ (case of the Er80 fiber), $H_{1}=0.9 \times\left(H_{1}+H_{2}\right), \quad \gamma=\delta=1.26 \times 10^{-11} \mathrm{~cm}^{3} \mathrm{~s}^{-1}$, $\beta_{1}=1.24 \times 10^{-11} \mathrm{~cm}^{3} \mathrm{~s}^{-1}, \quad \beta_{2}=1.26 \times 10^{-13} \mathrm{~cm}^{3} \mathrm{~s}^{-1}$, and $\sigma_{\mathrm{PB}} N_{\mathrm{v}}=4 \times 10^{-7} \mathrm{~mW}^{-2} \mathrm{~s}^{-1}$. Orders of magnitude for trapping and recombination coefficients are consistent with those expected for trapping and recombination cross-sections $\left(10^{-21}\right.$ $-10^{-12} \mathrm{~cm}^{2}$ [21]) and for the thermal velocity of holes in silica (significantly lower than that of electrons which is usually estimated to $10^{7} \mathrm{~cm} \mathrm{~s}^{-1}$ ). They are also similar to those used in [22] where we applied a generalized model to germaniumdoped optical fibers. Given these values, the order of magnitude of $\sigma_{\mathrm{PB}} N_{\mathrm{v}}$ was determined to reproduce correctly the experimental time scale. We used the unique set of parameters above for all results in Fig. 9-12, to allow comparison between the various simulations. A qualitative agreement with data is actually always obtained, even if these parameters are varied across several decades. Dose rates in $\mathrm{krad} \mathrm{min}^{-1}$ have been converted into pair generation rate $g_{0}$ in $\mathrm{cm}^{-3} \mathrm{~s}^{-1}$ thanks to the silica pair generation efficiency $\mathrm{G} \sim 5.18 \times 10^{15}$ pairs $\mathrm{cm}^{-3}$ $\mathrm{krad}^{-1}$. Computed RIA is presented in terms of the proportion of $\mathrm{Er}^{3+}$ to $\mathrm{Er}^{2+}$ ion conversion given by the ratio $r=n / N$. With chosen parameters, this ratio can be converted into $\mathrm{dB} \mathrm{m}^{-1}$ according to $\mathrm{RIA}\left(\mathrm{dB} \mathrm{m}^{-1}\right)=\sigma_{\mathrm{CC}} \times 3 \times 10^{22} \times r / \ln (10)$, where $\sigma_{\mathrm{CC}}$ is the total optical absorption cross-section by color centers at $976 \mathrm{~nm}$. This cross-section is not known, but taking $\sigma_{\mathrm{CC}}=10^{-19} \mathrm{~cm}^{2}$ as a typical order of magnitude gives a correspondence of $13 \mathrm{~dB} \mathrm{~m}^{-1}$ for each per cent of $\mathrm{Er}^{3+}$ to $\mathrm{Er}^{2+}$ ion conversion.

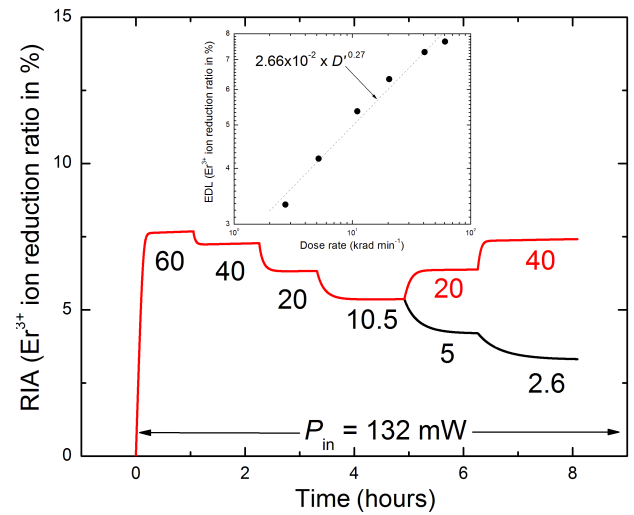

Fig. 9. Simulation of the first irradiation sequence of Fig. 2 (zone A) and a variant showing that EDLs given by the model are well reproducible for identical $\left(P_{\text {in }}, D^{\prime}\right)$ couples. Numeric plot labels: dose rates in $\mathrm{krad} \mathrm{min}^{-1}$. Inset: EDL as a function of the dose rate and its power law fit. 


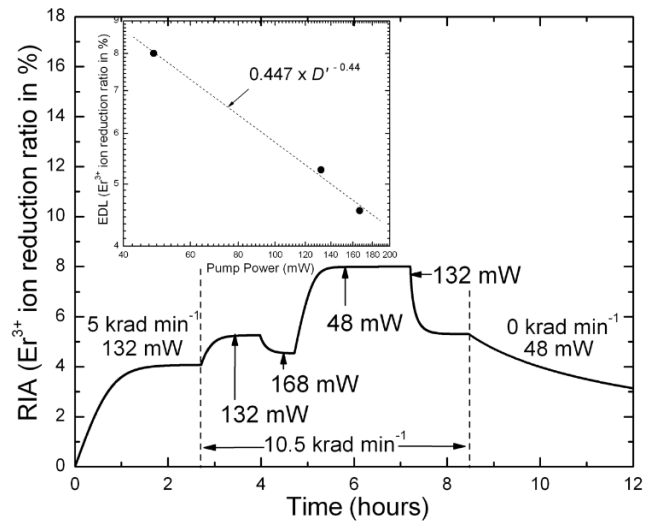

Fig. 10. Simulation of irradiation sequences of Fig. 2: end of zone A, zone B and beginning of zone $\mathrm{C}$ (dose rates and pump powers indicated in plots). Inset: EDL vs pump power at $10.5 \mathrm{krad} \mathrm{min}^{-1}$ and its power law fit.

Fig. 9 was obtained by simulating the first 6 steps of Fig. 2, zone A, with the same sequence of decreasing dose rates (from 60 down to $2.6 \mathrm{krad} \mathrm{min}^{-1}$ ). It also shows the result of a different series where the last two dose rates have been raised back to the third and second values to show that simulated RIA well returns to the same EDL at identical dose rates. Fig. 10 presents the simulation of the end of zone $\mathrm{A}$, zone $\mathrm{B}$, and of the beginning of zone $\mathrm{C}$ of Fig. 2. It mainly aims at showing that the modeled system behaves as real EDF when pump power switches are applied. As experimental RIA, the computed degradation well exhibits EDL that are only determined by the couple $\left(P_{\text {in }}, D^{\prime}\right)$. The power law dependence of the EDL on $D^{\prime}$ and $P_{\text {in }}$ are qualitatively reproduced (see insets of Fig. 9 and 10). Computed dose rate and pump exponents, $a=0.27$ and $b=-0.44$, are rather close to experimental ones for the Er80 fiber. Predicted conversion rates range between 5 to 10 percents. This order of magnitude is consistent with the 10 to $30 \%$ estimates, according to the glass composition, given in [21] (aluminum enhances conversion). In this reference, conversion ratios were obtained after a $300 \mathrm{krad}$ dose only, but in the absence of pump and hence of photo-induced mitigation.

Simulations of Fig. 11 have been done to check the model predictions with regards to zero-dose rate EDL. After irradiation at $10.5 \mathrm{krad} \mathrm{min}^{-1}$ under $132 \mathrm{~mW}$ pumping to reach a significant RIA level, the system was left to the sole action of the pump at $48.2,132$ and $178.5 \mathrm{~mW}$ to make it tend to the zero-dose rate EDL. As observed in experiments, this EDL is not zero: deep hole traps well introduce a non-bleachable RIA background. The second pump power switch (from 132 to $178.5 \mathrm{~mW}$ ) happens when the EDL is already established, and it does not induce any further bleaching. This point is in full agreement with the behavior highlighted in Fig. 3, region $\mathrm{C}$ (around $117 \mathrm{~h}$, same powers involved). Modeled mechanisms are therefore sufficient to account for the independence of zero-dose rate EDL on the pump power. In Fig. 11, the EDL could seem to be higher under $48.2 \mathrm{~mW}$ than under 132 or $178.5 \mathrm{~mW}$ pumping. In fact the RIA always falls to the same limit, the decay is just much slower at $48.2 \mathrm{~mW}$ and $\mathrm{PB}$ is markedly accelerated when the pump power is raised to 132 $\mathrm{mW}$. This may explain the apparent difference between the ill extrapolated zero-dose rate EDL marked in zones $\mathrm{A}$ and $\mathrm{C}$ of Fig. 2 at 13 and $18 \mathrm{~dB} \mathrm{~m}^{-1}$.

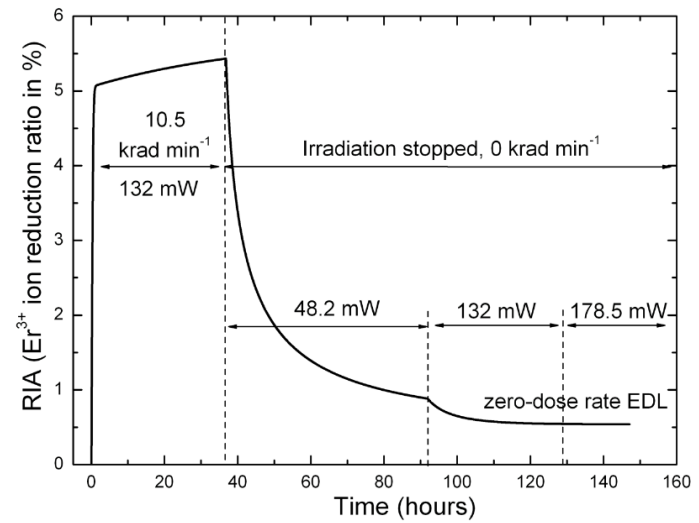

Fig. 11. RIA resulting from the simulation of zero-dose rate EDL after irradiation. Numeric labels: dose rates and pump powers.

\section{DISCUSSION AND CONCLUSION}

This work deals with RIA mitigation by $976 \mathrm{~nm}$ pumping in EDF. The underlying PB effect had been reported already [815] but not examined in detail. Proper characterization conditions using very short fibers samples have been proposed to liberate observables from spatial distribution effects and elucidate basic mechanisms. Our results demonstrate a novel and important property: due to pump-induced $\mathrm{PB}$, the local RIA can be tuned reversibly between equilibrium degradation levels (EDL) by varying the local pump power $P_{\text {in }}$ and/or the dose rate $D^{\prime}$ while the dose is being accumulated. EDL are found to vary as $D^{a}$ and $P_{\text {in }}{ }^{b}$, with $a=0.17$ to 0.34 and $b=-0.44$ to -0.26 for investigated EDF. Intermittent pumping also leads to the formation of mean EDL determined by pump power and operation duration. Basically, EDL could provide a most relevant reference to assess the radiation-resistance of EDF. They set the maximum (worst) local degradation level achievable at given $\left(P_{\mathrm{in},}, D^{\prime}\right)$. Optimizing EDL, rather than lower dose-dependent levels, focuses the discussion on 'rates' (pump energy rate and dose rate), while offering the best safety margin. These features had never been noticed to date because studies were based on standard fiber amplifier lengths. At fixed launched power and dose rate, regions of long EDF experiencing higher pump power (Fig. 1) reach their EDL rapidly, whereas RIA in lower pump power regions cannot stabilize readily due to their high EDL. This kinetics difference is reinforced with the passage of time, since lower pump power means faster RIA build-up, higher pump losses, even less efficient PB and enhanced EDL... Thus it takes much more time for length-integrated RIA to reach a stationary level, and this considerable delay probably explains why EDL have not been observed in long fibers. Local EDL increase as the fiber is exposed to irradiation, due to the development of pump power loss ( $P_{\text {in }}$ decreases). EDF regions with initially high (resp. low) pump power will experience more (resp. less) efficient PB so their RIA, together with their local EDL, will increase slowly (resp. rapidly). In other words, lower EDL are less rapidly damaged (i.e. raised). In spacebased applications, the small dose rate will not only make initial EDL low, but it will also ensure a very slow increase of this EDL as well as of the RIA towards it. Low EDL also require high pump power, but the notion of 'high' power is only relative to the dose rate magnitude. It is defined as a 
power inducing efficient $\mathrm{PB}$, i.e. capable of photo-ionizing color centers at a frequency comparable to that of their formation (set by $D^{\prime}$ '). Under low dose rate conditions, 'high' pump powers can be low actually. Therefore efficient $\mathrm{PB}$ and low EDL may be the case throughout the fiber, even if a simple one-directional pumping scheme is used. As a result, the low $976 \mathrm{~nm}$ RIA level predicted by the local model (Fig. 12) at a space dose rate can be probably projected for long fibers: EDFA should not be significantly damaged in space, whatever the total duration and dose (since EDL do not depend on the dose). This conclusion is opposite to that of enhanced low dose rate sensitivity (ELDRS) demonstrated in [10], which anticipates higher degradation levels in space than in accelerated tests made on Earth. Our experimental results did not exhibit ELDRS. However, we established that ELDRS is the most usual form of dose rate effects inherent in trappingrecombination models when detrapping is neglected [22]. The model of Fig. 8 is thus capable of yielding ELDRS if pump power and $\mathrm{PB}$ are weak and observation of ELDRS is not inconsistent with our model, depending on experimental conditions (small launched pump power and long fibers). Since we have now a validated local degradation model at our disposal, the next step is to implement it into EDFA simulations to take pump, ASE and signal distribution effects into account and develop predictive simulators for realistic EDFA. Then, we will be able to confirm the qualitative arguments developed in this conclusion and explore the conditions enabling ELDRS.

\section{REFERENCES}

[1] G.M. Williams, C.G. Atkins, E.J. Friebele, "Radiation effects in erbiumdoped optical fibers," Electron. Lett., Vol. 28, No 19, pp. 1816-1818, 1992.

[2] G.M. Williams, E.J. Friebele, "Space radiation effects on erbium-doped fiber devices: sources, amplifiers and passive measurements," IEEE Trans. Nucl. Sci., Vol. 45, No 3, pp. 1531-1536, 1998

[3] O. Berné, M. Caussanel, O. Gilard, "A model for the prediction of EDFA gain in a space radiation environment," IEEE Photon. Technol. Lett., Vol. 16, No 10, pp. 2227-2229, 2004

[4] M. Caussanel, O. Gilard, M. Sotom, P. Signoret, J. Gasiot, "Extrapolation of radiation-induced EDFA gain degradation at space dose rate," Electron. Lett., Vol. 41, No 4, pp. 168-170, 2005

[5] S. Girard, B. Tortech, E. Regnier, M. Van Uffelen, A. Gusarov, Y. Ouerdane, J. Baggio, P. Paillet, V. Ferlet-Cavrois, A. Boukenter, J-P. Meunier, F. Berghmans, J.R. Schwank, M.R. Shaneyfelt, J.A. Felix, E.W. Blackmore, H. Thienpont, "Proton- and gamma-induced effects on erbium-doped optical fibers," IEEE Trans. Nucl. Sci., Vol. 54, No 6, pp. 2426-2434, 2007

[6] B. Tortech, S. Girard, E. Regnier, Y. Ouerdane, A. Boukenter, J-P. Meunier, M. Van Uffelen, A. Gusarov, F. Berghmans, H. Thienpont, "Core versus cladding effects of proton irradiation on erbium-doped optical fiber: micro-luminescence study," IEEE Trans. Nucl. Sci., Vol. 55, No 4, pp. 2223-2228, 2008

[7] K.V. Zotov, M.E. Likhachev, A.L. Thomashuk, M.M Bubnov, M.V. Yashkov, A.N. Guryanov, S.N. Klyamkin, "Radiation-resistant erbiumdoped fiber for spacecraft applications," IEEE Trans. Nucl. Sci., Vol. 55, No 4, 2008

[8] K.V. Zotov, M.E. Likhachev, A.L. Thomashuk, A.F. Kosolapov, M.M Bubnov, M.V. Yashkov, A.N. Guryanov, E.M. Dianov, "Radiationresistant Er-doped fibers: optimization of pump wavelength," IEEE Photon. Technol. Lett., Vol. 20, No 17, 2008

[9] M. Lezius, K. Predehl, W. Stöwer, A. Türler, M. Greiter, Ch. Hoeschen, P. Thirolf. W. Assman, D. Habs, C. Ekström, T.W. Hänsch, R. Holzwarth, "Radiation-induced absorption in rare earth doped optical fibers," IEEE Trans. Nucl. Sci., Vol. 59, No 2, pp. 425-433, 2012.

[10] O. Gilard, J. Thomas, L. Trousselier, M. Myara, P. Signoret, E. Burov, M. Sotom, "Theoretical explanation of enhanced low dose rate sensitivity in erbium-doped optical fibers," Appl. Opt., Vol. 51, No 13, pp. 2230-2235, 2012.

[11] J. Thomas, M. Myara, L. Trousselier, E. Burov, A. Pastouret, D. Boivin, G. Mélin, O. Gilard, M. Sotom, P. Signoret, "Radiation-resistant erbiumdoped-nanoparticles optical fiber for space applications," Opt. Express, Vol. 20, No 3, pp. 2435-2444, 2012

[12] S. Girard, M. Vivona, A. Laurent, B. Cadier, C. Marcandella, T. Robin, E. Pinsard, A. Boukenter, Y. Ouerdane, "Radiation hardening techniques for $\mathrm{Er} / \mathrm{Yb}$ doped optical fibers and amplifiers for space applications," Opt. Express, Vol. 20, No 8, pp. 8457-8465, 2012

[13] S. Girard, A. Laurent, E. Pinsard, T. Robin, B. Cadier, M. Boutillier, C. Marcandella, A. Boukenter, Y. Ouerdane, "Radiation-hard erbium optical fiber and fiber amplifier for both low- and high-dose missions," Opt. Lett., Vol. 39, No 9, pp. 2541-2544, 2014

[14] E.J. Friebele, "Radiation protection of fiber optic materials: effects of cerium doping on the radiation-induced absorption," Appl. Phys. Lett., Vol. 27, pp. 210-212, 1975

[15] T-S. Peng, Y-W. Huang, L.A. Wang, R-Y. Liu, F-I. Chou, "Photoannealing effects for erbium doped fiber sources after gamma irradiation tests by using $532 \mathrm{~nm}$ and $976 \mathrm{~nm}$ lasers," Proc. of SPIE, Vol. 7503, 750375,2009

[16] J-B. Duchez, F. Mady, Y. Mebrouk, N. Ollier, M. Benabdesselam, "Interplay between photo- and radiation-induced darkening in ytterbiumdoped fibers," Opt. Lett., Vol. 39, No 20, pp. 5969-5972, 2014.

[17] D.L. Griscom, M.E. Gingerich, E.J. Friebele, "Radiation-induced defects in glasses: origin of power-lax dependence of concentration on dose," Phys. Rev. Lett., Vol. 71, No 7, pp. 1019-1022, 1993

[18] F. Mady, J-B. Duchez, Y. Mebrouk, M. Benabdesselam, "A physical model of the photo- and radiation-induced degradation of ytterbiumdoped silica optical fibers," AIP Conf. Proc., 1624, 87, 2014.

[19] F. Mady, M. Benabdesselam, W. Blanc, "Thermoluminescence characterization of traps involved in the photodarkening of ytterbiumdoped silice fibers," Opt. Lett., Vol. 35, No 21, pp. 3541-3543, 2010.

[20] Y. Mebrouk, F. Mady, M. Benabdesselam, J-B Duchez, W. Blanc, "Experimental evidence of $\mathrm{Er}^{3+}$ ion reduction in the radiation-induced degradation of erbium-doped silica fibers," Opt. Lett., Vol. 39, No 21, pp. 6154-6157, 2014.

[21] D.J. DiMaria, "Defects and impurities in thermal $\mathrm{SiO}_{2}$ ", in The Physics of $\mathrm{SiO}_{2}$ and its Interfaces, Elsevier, 1978, pp. 160-178.

[22] F. Mady, M. Benabdesselam, J-B. Duchez, Y. Mebrouk, S. Girard, "Golbal view on dose rate effects in silica-based fibers and devices damaged by radiation-induced carrier trapping," IEEE Trans. Nucl. Sci., Vol. 60, No 6, pp. 4341-4348, 2013. 